13

\title{
Стабильность полевой эмиссии одиночной углеродной нанотрубки
}

\author{
(C) С.В. Булярскийㅁ, А.А. Дудин, ${ }^{1}$ А.В. Лакалин, ${ }^{1,2,}$ А.П. Орлов, ${ }^{1}$ А.А. Павлов, ${ }^{1}$ \\ P.М. Рязанов, ${ }^{3}$ A.A. Шаманаев ${ }^{3}$ \\ ${ }^{1}$ Институт нанотехнологий микроэлектроники РАН, \\ 119991 Москва, Россия \\ ${ }^{2}$ Ульяновский государственный университет, \\ 432017 Ульяновск, Россия \\ ${ }^{3}$ Научно-производственный комплекс „Технологический центр“, \\ 124498 Москва, Зеленоград, Россия \\ ؟ e-mail: a.v.lakalin@mail.ru
}

(Поступило в Редакцию 1 августа 2017 г. В окончательной редакции 17 октября 2017 г.)

\begin{abstract}
Экспериментально наблюдался разогрев одиночной многостенной углеродной нанотрубки при протекании через нее тока полевой эмиссии и появление термоэмиссионной составляющей. Нагрев нанотрубки при протекании тока происходит в результате выделения джоулевой теплоты на ее последовательном сопротивлении. Из решения уравнения теплопроводности была сделана оценка величины температуры перегрева эмитирующего конца. Установлены условия устойчивости полевой эмиссии и возникновения термополевой эмиссии.
\end{abstract}

DOI: 10.21883/JTF.2018.06.46026.2447

\section{Введение}

Одним из важных физических явлений в углеродных нанотрубках (УНТ), которое имеет перспективу практического применения, является полевая эмиссия [1]. Достаточно полно исследована физика данного явления, а также разработаны технологии эмиссионных структур. Перспективы использования полевой эмиссии из УНТ связаны с тем, что диаметр нанотрубок много меньше их длины, тем самым они обладают большим аспектным отношением, которое позволяет достигать значительного усиления электрического поля у конца нанотрубки, эмитирующей электроны. Поэтому не удивительно, что в 1995 г. появилось несколько работ, в которых описывалось явление полевой эмиссии из УНТ [2-4]. Это явление легло в основу ряда важных с точки зрения практики применений: плоских экранов мониторов [5,6], миниатюрных рентгеновских трубок [7,8], светоизлучающих устройств $[9,10]$, миниатюрных вакуумных ламп $[11,12]$, усилителей терагерцового диапазона [13,14], высокочастотных вакуумных переключателей [15].

Широкие перспективы применения полевой эмиссии (ПЭ) требуют детального изучения этого явления, в первую очередь с точки зрения получения высоких плотностей тока эмиссии и стабильности этого процесса $[16,17]$. Вольт-амперная характеристика холодного катода на участке преобладания тока полевой эмиссии, как правило, описывается формулой ФаулераНордгейма. Подробный анализ этой модели и переход ее в область термоэлектронной эмиссии выполнен в работах $[15,18]$. Расчеты тока полевой эмиссии для углеродных нанотрубок приведены, например, в [19]. Сама зависимость Фаулера-Нордгейма в удобной для использования форме имеет вид [20]

$$
J=\frac{e^{3} E^{2}}{8 \pi h \varphi t^{2}(y)} \exp \left(-\frac{8 \pi \sqrt{2 m} \varphi^{3 / 2} \theta(y)}{3 h e E}\right),
$$

где $t(y)=1+0.1107 y^{1.33}, \theta(y)=1-y^{1.69}, y=\frac{e}{\varphi} \sqrt{\frac{e E}{4 \pi \varepsilon_{0}}}$, $e$ - элементарный заряд (С), $m$ - масса свободного электрона $(\mathrm{kg}), h$ - постоянная Планка (Js), $\varepsilon_{0}$ электрическая постоянная $(\mathrm{F} / \mathrm{m}), E-$ напряженность локального электрического поля вблизи эмитирующей поверхности $(\mathrm{V} / \mathrm{m}), \varphi$ - работа выхода электрона $(\mathrm{J})$, $J$ - плотность тока ПЭ $\left(\mathrm{A} / \mathrm{m}^{2}\right), t(y)$ и $\theta(y)$ - слабо изменяющиеся функции, которые при сопоставлении формулы (1) с экспериментальными данными могут быть приняты равными единице без увеличения погрешности в определении работы выхода. Если пренебречь слабой степенной зависимостью функций $t(y)$ и $\theta(y)$, положив $t(y) \approx 1$ и $\theta(y) \approx 1$, то после подстановки всех констант и перевода работы выхода в $\mathrm{eV}$ получается выражение

$$
J=1.54 \cdot 10^{-6} \frac{E^{2}}{\varphi} \exp \left(-\frac{6.83 \cdot 10^{9} \varphi^{3 / 2}}{E}\right),
$$

в котором $[\varphi]=\mathrm{eV},[E]=\mathrm{V} / \mathrm{m},[J]=\mathrm{A} / \mathrm{m}^{2}$.

Уже в первых работах, посвященных изучению явления полевой эмиссии, было обнаружено, что конец нанотрубки разогревается в процессе протекания эмиссионного тока, а его температура пропорциональна квадрату плотности тока $[19,21]$, что находится в соответствии с законом Джоуля-Ленца. Разогрев конца нанотрубки ведет к появлению токов термоэлектронной эмиссии (ТЭ), которые могут быть большими при высоких плотностях тока и даже превосходить токи полевой эмиссии. Кроме 
того, нанотрубка обладает определенным сопротивлением, которое дает определенный вклад в вид вольтамперной характеристики, принимая при больших плотностях тока часть напряжения на себя. Таким образом, существование сопротивления у нанотрубки приводит к отклонению экспериментальных результатов от формулы (1), а появление токов ТЭ - к нестабильности вольтамперных характеристик. Источником нестабильности эмиссионных токов также может быть изменение работы выхода [22,23]. Однако применительно к УНТ этот фактор требует дополнительного исследования.

В настоящей работе экспериментально исследованы эмиссионные токи одиночной многостенной нанотрубки в широком диапазоне значений тока, выявлены отклонения вольт-амперной характеристики от зависимости Фаулера-Нордгейма, проведен расчет температуры разогрева нанотрубки, проанализированы условия, при которых сопротивление нанотрубки и ток термоэлектронной эмиссии оказывают существенное влияние на вид вольт-амперной характеристики, а также приведен алгоритм расчета параметров углеродной нанотрубки: электрического сопротивления, зависимости температуры разогрева нанотрубки от величины тока.

\section{Экспериментальные результаты}

Углеродные нанотрубки выращивались методом химического осаждения (CVD) из газовой фазы в установке Plasmalab System 100 фирмы Oxford Instruments на кремниевой подложке, на которую был нанесен катализатор, состоящий из двуслойной металлической пленки: титан $-10 \mathrm{~nm}$ и никель $-2 \mathrm{~nm}$. Катализатор покрывался оксидом кремния, в котором вскрывались окна диаметром $0.7 \mu \mathrm{m}$. Рост УНТ осуществлялся в режиме CVD в потоке ацетилена с добавлением аммиака в отношении $3: 1$. Величина газового потока поддерживалась постоянной во время роста. Температура синтеза составляла $600^{\circ} \mathrm{C}$. Результатом роста являлись одиночные многостенные углеродные нанотрубки высотой $2-3 \mu \mathrm{m}$ (рис. 1).

Измерения проводили в высоком вакууме в камере двулучевой системы FEI Helios NanoLab 650i. Измерительный электронный микроскоп позволяет получать изображения с разрешением не хуже $0.7 \mathrm{~nm}$ при ускоряющем напряжении не более $1 \mathrm{kV}$. Рабочее давление в измерительной камере составляло $5 \cdot 10^{-5} \mathrm{~Pa}$. В этой камере находилась зондовая система Kleindiek Nanotechnik с 4 отдельными независимыми манипуляторами. Для измерений вольт-амперных характеристик на постоянных токах был использован программируемый двухканальный источник-измеритель Source Meter 2634В фирмы Keithley. Данный прибор оптимизирован для измерений слаботочных сигналов вплоть до $10^{-15}$ А и оснащен специальными экранированными триаксиальными выводами с функцией компенсации сверхмалых токов. Входное сопротивление прибора (более $100 \mathrm{~T} \Omega$ ) обеспечивает при

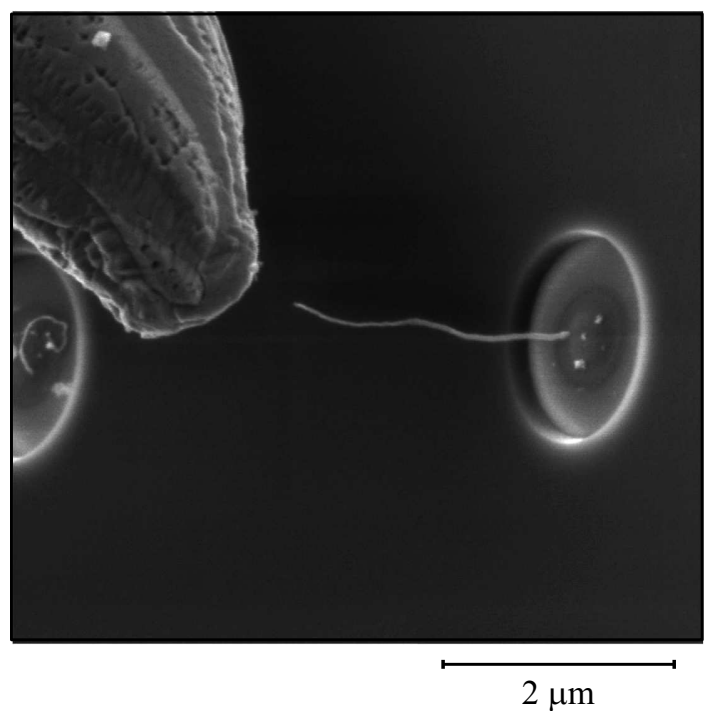

Рис. 1. Фотография исследуемой эмиссионной системы.

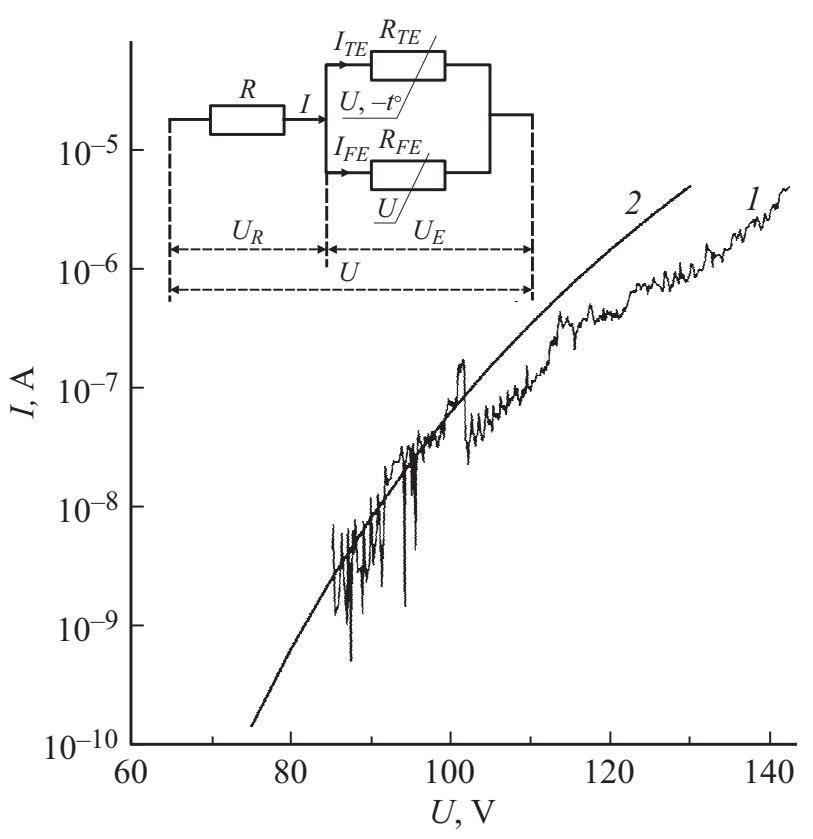

Рис. 2. Эмиссионные токи исследуемой УНТ: 1 - экспериментальная ВАХ, 2 - рассчитанный ток полевой эмиссии $(\varphi=4.8 \mathrm{eV})$. На вставке - эквивалентная электрическая схема УНТ.

измерениях минимальный уровень вносимых искажений и ошибок в тестируемые схемы в этом классе приборов. Вольт-амперная характеристика эмиссионного тока одиночной многостенной нанотрубки приведена на рис. 2. Она являлась исходной для дальнейшей обработки.

На вставке к рис. 2 приведена электрическая схема цепи, в которой протекает эмиссионный ток. Этот ток складывается из двух составляющих: полевой и термоэлектронной, причем каждый процесс характеризуется своим нелинейным сопротивлением $\left(R_{F E}\right.$ и $\left.R_{T E}\right)$. Общее 
напряжение, приложенное к цепи $(U)$, складывается из суммы напряжений, одно из которых падает на сопротивлении нанотрубки $\left(U_{R}\right)$, а другое - на нелинейном сопротивлении эмитирующего конца нанотрубки $\left(U_{E}\right)$. Данная схема диктует следующую последовательность действий: 1) рассчитывается ток полевой эмиссии (расчет приведен в [24]). При этом величина работы выхода подбирается таким образом, чтобы ток полевой эмиссии совпадал с начальным участком экспериментальной вольт-амперной характеристики (рис. 2). 2) отклонение эксперимента от расчета обусловлено падением напряжения на углеродной нанотрубке. Это падение напряжения вычисляется как разность между экспериментом и расчетом эмиссионного тока, что дает вольт-амперную характеристику самой углеродной нанотрубки и возможность определения ее сопротивления. Преобразованная характеристика получается вычитанием из общей вольтамперной характеристики падения напряжения на ее сопротивлении (рис. 3). На данном рисунке видно, что имеет место появление участка с отрицательным сопротивлением, которое обусловлено разогревом конца нанотрубки и действием явления термоэлектронной эмиссии. Таким образом, при разогреве конца трубки до некоторой критической температуры появляется ток термоэлектронной эмиссии, напряжение на эмитирующем конце $\left(U_{E}\right)$ падает. Вместе с этим падает ток полевой эмиссии (кривая 2). Поэтому при увеличении плотности тока его термоэлектронная составляющая доминирует и продолжается разогрев конца нанотрубки. Температура конца трубки растет и может достигнуть нескольких тысяч градусов. Такая температура нанотрубки приводит к ее разрушению, и эмиссионный ток деградирует. Само явление деградации связано с перегревом нанотрубки. Ниже проводятся необходимые расчеты, чтобы выявить условия, при которых эмиссионный процесс будет стабильным.

\section{Расчет температуры разогрева конца одиночной углеродной нанотрубки}

Нанотрубка разогревается при протекании в ней электрического тока. Ее температура не является одинаковой на двух ее концах. Предполагается, что конец нанотрубки, который контактирует с подложкой, имеет ту же температуру, что и сама подложка, т. е. температуру окружающей среды. Температура противоположного конца была вычислена в результате решения уравнения теплопроводности с учетом радиационного охлаждения и выделения тепла, которое обусловлено протеканием тока $[21,25]$ :

$S \frac{d}{d x}\left(\lambda(T) \frac{d T}{d x}\right) d x-2 \pi r \eta \sigma\left(T^{4}-T_{0}^{4}\right) d x+I^{2} \frac{R(T)}{L} d x=0$,

где $S=\pi\left(r^{2}-r_{0}^{2}\right)$ - площадь поперечного сечения УНТ, $r$ - внешний радиус УНТ, $r_{0}-$ внутренний

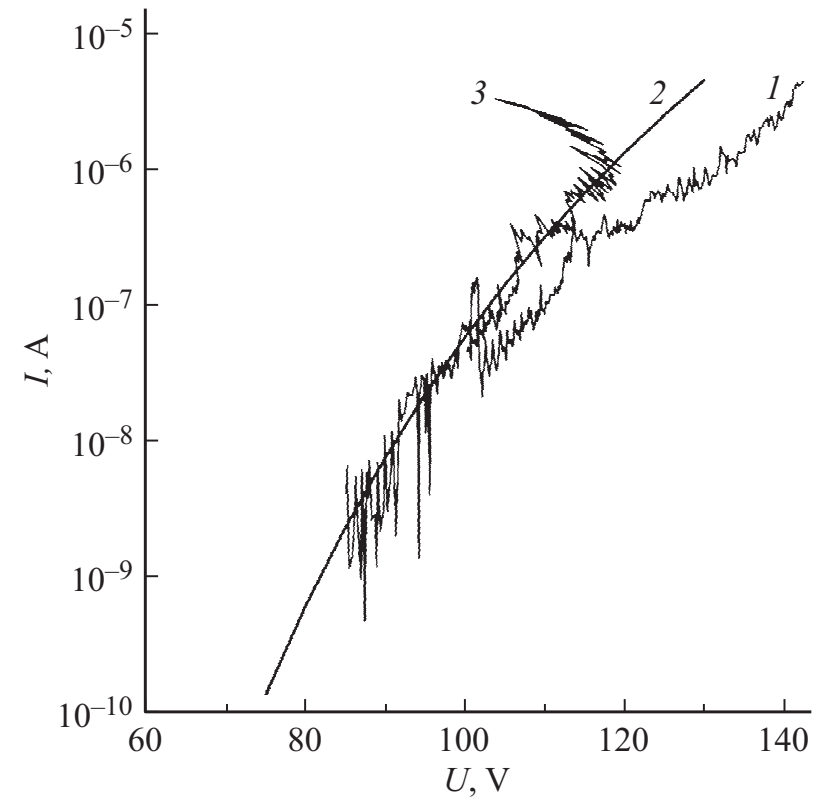

Рис. 3. Эмиссионные токи исследуемой УНТ: 1 - экспериментальная ВАХ, 2 - рассчитанный ток полевой эмиссии $(\varphi=4.8 \mathrm{eV}), 3$ - сумма токов полевой и термоэлектронной эмиссий.

радиус УНТ, $\lambda(T)-$ коэффициент теплопроводности вдоль оси УНТ, $T=T(x)$ - температура вдоль оси УНТ, $T_{0}$ — температура окружающих тел (подложки), $L-$ длина УНТ, $R(t) / L$ - электрическое сопротивление единицы длины УНТ, $\eta$ - коэффициент серости теплового излучения УНТ $(\eta<1)$ в нашем случае принимался равным 0.9, $\sigma$ - постоянная Стефана-Больцмана, $I$ - ток, протекающий через УНТ (эмиссионный ток). Граничные условия для уравнения (3) имеют вид

$$
T(0)=T_{0}, \quad \frac{d T(L)}{d x}=0
$$

В работе [21] было получено аналитическое решение уравнения (3) в случае отсутствия радиационного охлаждения и при условии, что коэффициент теплопроводности $\lambda$, а также сопротивление $R$ нанотрубки не зависят от температуры. Однако для УНТ имеет место температурная зависимость $\lambda$ и $R$, поэтому результаты работы [21] нужно рассматривать как приближенные. В работе [25] предполагалось, что коэффициент теплопроводности $\lambda$ и сопротивление $R$ описываются степенными функциями от температуры. Для случая $\lambda=a T^{3}, R=b T^{4}+c$ в [25] получено аналитическое решение уравнения (3). В работе [26] предполагалось, что $\lambda=\lambda_{0}\left(T / T_{0}\right)^{\alpha}, R=R_{0}\left(T / T_{0}\right)^{\alpha}$, где $\alpha-$ подгоночный параметр, а уравнение (3) решалось численно.

Однако степенная зависимость вида $\lambda=C T^{\alpha}$ для коэффициента теплопроводности имеет место только при температурах, ниже характеристической температуры Дебая [27]. При высоких температурах в силу ангармонизма длинноволновых колебаний и других причин 


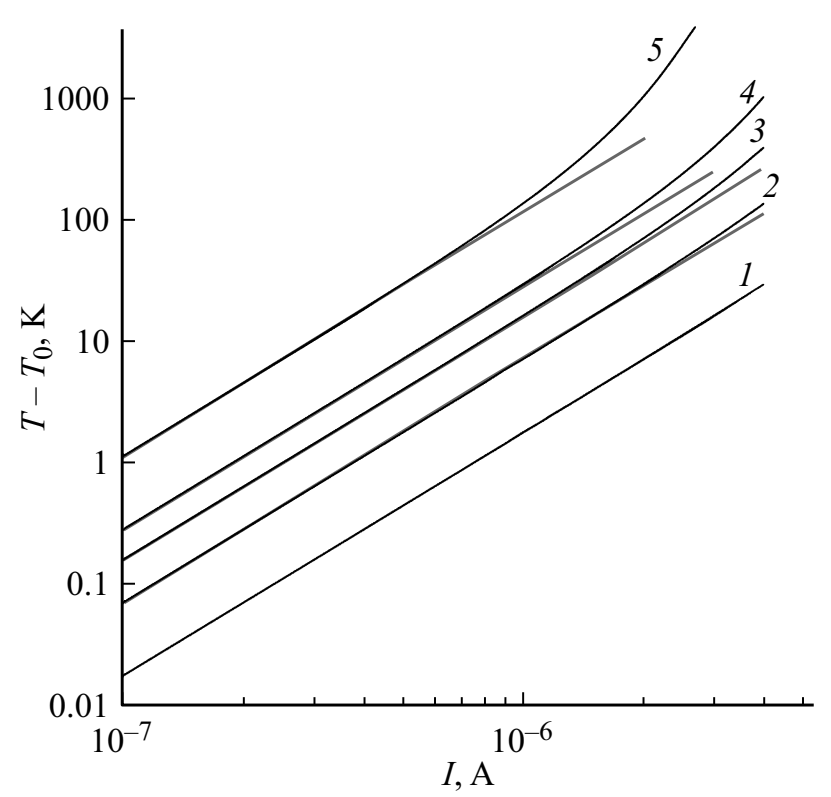

Рис. 4. Зависимость температуры перегрева эмитирующего конца УНТ от протекающего тока для УНТ разной длины: $1-0.5 \mu \mathrm{m}, 2-1.0 \mu \mathrm{m}, 3-1.5 \mu \mathrm{m}, 4-2.0 \mu \mathrm{m}, 5-4.0 \mu \mathrm{m}$.

теплопроводность твердого тела убывает по закону $1 / T$, а именно [27]

$$
\lambda=\lambda_{0} \frac{\Theta}{T},
$$

где $\Theta-$ температура Дебая. Энергия фонона Дебая углеродных нанотрубок равна $0.103 \mathrm{eV}$ [28]. Соответственно температура Дебая $\Theta=1190 \mathrm{~K}$. Именно эта зависимость доминирует в области высоких температур, когда возможно возникновение термоэлектронной эмиссии. Поэтому для расчетов разогрева нанотрубки была выбрана модель (5).

Для оценки температуры эмитирующего конца УНТ численно решалось уравнение теплопроводности (3) с граничными условиями (4) с температурной зависимостью коэффициента теплопроводности вида (5). Значения параметров были следующими: $\lambda_{0}=140 \mathrm{~W} /(\mathrm{m} \cdot \mathrm{K})$, $\Theta=1190 \mathrm{~K}$. Внешний $(r=20 \mathrm{~nm})$ и внутренний радиусы $\left(r_{0}=15 \mathrm{~nm}\right)$ определялись в результате измерения с помощью просвечивающего электронного микроскопа. Длина нанотрубки при расчетах температуры изменялась от 0.5 до $4 \mu \mathrm{m}$. Температура холодного конца нанотрубки, контактирующего с подложкой, принималась равной $T_{0}=300 \mathrm{~K}$. При выбранных значениях $\lambda_{0}$ и $\Theta$ в интервале температур $200-3000 \mathrm{~K}$ коэффициент теплопроводности $\lambda$ лежит в пределах $830-55 \mathrm{~W} /(\mathrm{m} \cdot \mathrm{K})$. Это соответствует литературным данным, согласно которым $\lambda$ может изменяться от 3000 до $25 \mathrm{~W} /(\mathrm{m} \cdot \mathrm{K})$ [25].

Уравнение (3) решалось для различных значений тока $I$, и тем самым получалась зависимость $T=f(I)$, где $T$ - температура эмитирующего конца (рис. 4).

На этом рисунке проведено сопоставление точного решения уравнения (3) с приближенным аналитиче- ским решением, полученным при условии отсутствия радиационного охлаждения и постоянства коэффициента теплопроводности:

$$
T(L)-T_{0}=\frac{I^{2} R}{2 \lambda S} L .
$$

Упрощенное решение является справедливым при малых температурах перегрева конца нанотрубки, когда перегрев не превышает $100 \mathrm{~K}$. Точное численное решение должно быть использовано, если перегрев превышает эту величину. Различие результатов этих двух подходов в первую очередь определяется температурной зависимостью коэффициента теплопроводности (5). Этот коэффициент стоит в знаменателе формулы для расчета температуры перегрева. Когда он уменьшается, перегрев увеличивается. Температура перегрева растет пропорционально квадрату тока эмиссии. Эта величина становится значительной при токах через одиночную нанотрубку порядка единиц $\mu \mathrm{A}$. В этой области могут появляться токи термоэлектронной эмиссии.

\section{Обсуждение результатов}

Электрическая схема протекания токов представлена на рис. 2. В соответствии с этой схемой ток эмиссии складывается из полевой и термоэлектронной составляющих. Этот ток представлен кривой 3 на рис. 3 . Ток полевой эмиссии представлен на этом рисунке кривой 2. Очевидно, что при максимальных значениях напряжения, при котором начинается участок отрицательного сопротивления, токи полевой и термоэлектронной эмиссии примерно равны. Дальнейший рост тока обусловлен термоэлектронной составляющей, а ток полевой эмиссии уменьшается, изменяясь при этом по кривой 2.

Таким образом, участок отрицательного сопротивление вольт-амперной характеристики (рис. 3) обусловлен тем, что ток термоэлектронной эмиссии преобладает над полевым током. При этом конец нанотрубки уже перегрет до такой степени, что она может разрушаться. Напряжение и ток, при котором начинается участок отрицательного сопротивления, можно считать граничными. Как только суммарный ток превышает это значение, эмиссия становится нестабильной и начинаются процессы деградации.

Важно провести оценку условий, при которых возможна деградация эмиссионных токов. Существует условная граница тока, превышение которой вызывает быстрый перегрев конца нанотрубки и деградацию эмиссии. На самой границе полный ток равен сумме токов полевой и термоэлектронной эмиссий, $I=I_{F E}+I_{T E}$. В дальнейшем преобладает термоэлектронный ток. Поэтому в качестве условия смены механизма эмиссии можно выбрать равенство токов $I_{T E}=I_{F E}$ или $I_{T E}(T)=I / 2$. Это условие позволяет оценить геометрические размеры углеродных 


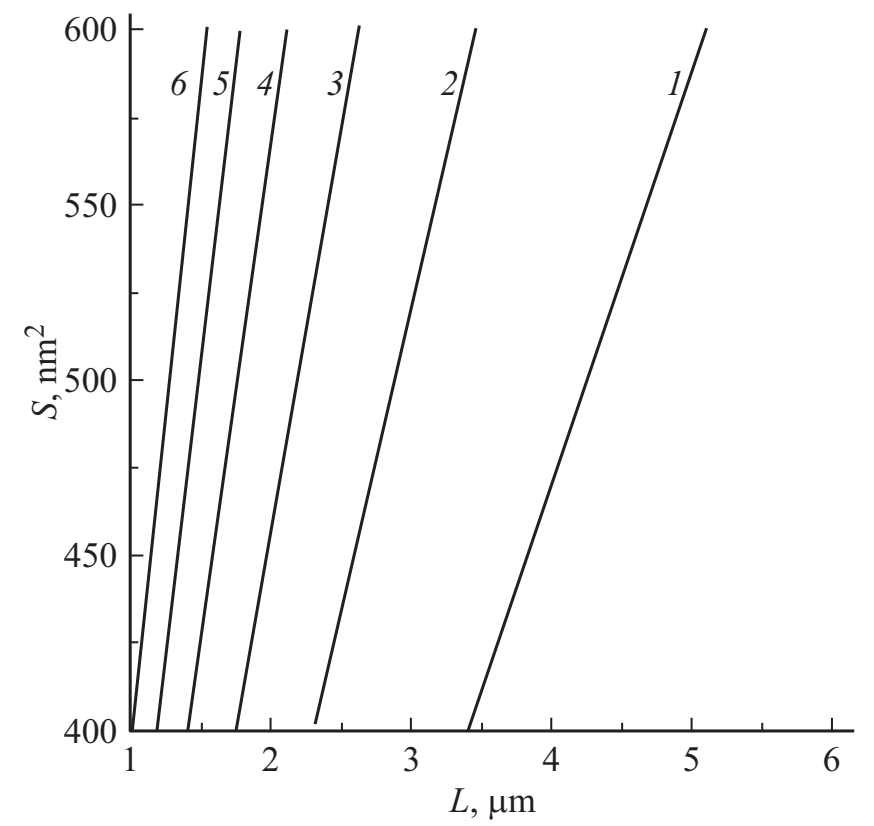

Рис. 5. Семейство зависимостей $S(L)$, для которых $I_{T E}=I / 2$. Значения тока $I: 1-2 \mu \mathrm{A}, 2-3 \mu \mathrm{A}, 3-4 \mu \mathrm{A}, 4-5 \mu \mathrm{A}$, $5-6 \mu \mathrm{A}, 6-7 \mu \mathrm{A}$.

УНТ катода при фиксированном значении протекающего тока, при которых начинается их разогрев.

Температура разогрева конца УНТ определяется током термоэлектронной эмиссии $\left(I_{T E}\right)$ и геометрическими размерами нанотрубки, которые в конечном итоге определяют и величину ее электрического сопротивления (6). Для приблизительной оценки температуры перегрева достаточно ограничиться формулой (6), которая, как показывают результаты точного расчета, приведенные на рис. 4, являются хорошей аппроксимацией. Сопротивление нанотрубки оценим по формулам:

$$
R=\rho \frac{L}{S}, \quad \rho=R_{0} \frac{S_{0}}{L_{0}} .
$$

Из экспериментов было определено $R_{0}=10 \mathrm{M} \Omega$, $S_{0}=5.5 \cdot 10^{2} \mathrm{~nm}^{2}, \quad L_{0}=2.36 \mu \mathrm{m} \quad$ и вычислено $\rho=$ $=2.33 \cdot 10^{-3} \Omega \cdot \mathrm{m}$. Выражение для тока термоэлектронной эмиссии имеет вид

$$
I_{T E}=S_{C N T} \frac{4 \pi m_{n}^{*}}{h^{3}}(k T)^{2} \exp \left[-\frac{\varphi}{k T}\right],
$$

где $S_{C N T}=2 \pi r^{2}$ - площадь эмитирующей поверхности УНТ (площадь поверхности полусферы), $m_{n}^{*}$ - эффективная масса электрона в УНТ, $k$ - постоянная Больцмана, $T$ - абсолютная температура. В выражении (8) не учитывается понижение высоты барьера электрическим полем (эффект Шоттки).
Условная граница перехода стабильного процесса в нестабильный определяется из формул (6) и (8)

$$
\frac{1}{2} I=S_{C N T} A(T(L))^{2} \exp \left[-\frac{\varphi}{k T(L)}\right] \text {. }
$$

Подставляя (6) в (9), получаем

$$
\frac{I}{S_{C N T}}=2 A\left(T_{0}+\frac{I^{2} \rho L^{2}}{2 \lambda S^{2}}\right)^{2} \exp \left[-\frac{\varphi}{k\left(T_{0}+\frac{I^{2} \rho L^{2}}{2 \lambda S^{2}}\right)}\right] \text {. }
$$

Формула (10) позволяет для УНТ заданного радиуса $r$ вычислить площадь сечения $S$ и длину $L$, которые соответствуют началу появления тока термоэлектронной эмиссии $\left(T_{T E}\right)$ при заданном значении полного тока $I$ (рис. 5). Этот рисунок представляет некоторые области в пространстве длина - площадь сечения УНТ. Это пространство разделено на области кривыми, которые рассчитаны для определенных токов. Данные кривые отражают границу, за которой наступает режим термоэлектронной эмиссии и деградации УНТ. Для каждой кривой справедливо утверждение: если длина нанотрубки больше, а площадь меньше, то мы переходим границу и попадаем в область деградации. При обратных соотношениях между параметрами, а именно длина меньше граничной, а площадь больше, попадаем в область стабильности эмиссии.

\section{Заключение}

Изучение эмиссионных процессов с одиночной нанотрубки показало, что при увеличении тока происходит разогрев эмитирующего электроны конца. В этом случае наряду с током полевой эмиссии появляется ток термоэлектронной эмиссии. С увеличением тока температура перегрева увеличивается, ток определяется практически только термоэлектронной эмиссией. При этом сам процесс эмиссии становится нестабильным. Так, температура перегрева может превысить $2000^{\circ} \mathrm{C}$, и нанотрубка начинает разрушаться.

Для анализа этих процессов в работе разработан алгоритм, который опирается на численные расчеты тока полевой эмиссии и температуры перегрева. Показано, что при перегреве возникает участок отрицательного сопротивления. В этот момент начинает преобладать термоэлектронный ток. Вычислена связь между величиной тока, длиной и площадью поперечного сечения нанотрубки, которая позволяет оценить области, в которых могут развиваться деградации катода и эмиссия становиться неустойчивой.

Работа выполнена при поддержке Министерства образования и науки РФ в рамках программы Государственной поддержки научных исследований, а также при поддержке Минобрнауки России (проект № 16.9007.2017/БЧ). 


\section{Список литературы}

[1] Булярский C.B. Углеродные нанотрубки. Технология. Управление свойствами. Применение. Ульяновск: Стрежень, 2011. 479 с.

[2] Chernozatonskii L.A., Gulyaev Y.V., Kosakovskaja Z.J., Sinitsyn N.I., Torgashov G.V., Zakharchenko Yu.F., Fedorov E.A., Val'chuk V.P. // Chem. Phys. Lett. 1995. Vol. 233. P. 63-68.

[3] De Heer W.A., Chatelain A., Ugarte D. // Science. 1995. Vol. 270. P. 1179-1180.

[4] Rinzler A.G., Hafner J.H., Nikolaev P., Nordlander P., Colbert D.T., Smalley R.E., Lou L., Kim S.G. // Science. 1995. Vol. 269. P. 1550-1553.

[5] Wang Q.H., Yan M., Chang R.P.H. // Appl. Phys. Lett. 2001. Vol. 78. P. 1294-1296.

[6] Mauger M., Vu T.V. // J. Vac. Sci. Technol. B. 2006. Vol. 24. P. 997-1003.

[7] Reyes-Mena A., Jensen Ch., Bard E., Turner D., Erdmann K.G. // Adv. in X-ray Analys. 2005. Vol. 48. P. 204-209.

[8] Matsumoto T., Mimura H. // Appl. Phys. Lett. 2003. Vol. 82. P. 1637-1639.

[9] Saito Y., Uemura S., Hamaguchi K. // Jpn. J. Appl. Phys. 1998. Vol. 37. P. L346-L348.

[10] Croci M., Arfaoui I., Stöckli T., Chatelain A., Bonard J.-M. // Microelectron. J. 2004. Vol. 35. P. 329-336.

[11] Yasutomo Y., Ohue W., Gotoh Y, Tsuji H. // IEEE International Meeting Future of Electron Devices. Kansai, 2012.

[12] Sabaut L., Ponard P., Mazellier J.-P., Legagneux P. // J. Vac. Sci. Technol. B. 2016. Vol. 34. P. 2.

[13] Yuan X., Zhu W., Zhang Y., Xu N., Yan Y., Wu J., Shen Y., Chen J., She J. // Sci. Rep. 6. 2016. Art. num. 32936.

[14] Paoloni C., Carlo A., Brunetti F., Mineo M. // Terahertz Sci. Technol. 2011. Vol. 4., N 4. P. 1102-1110.

[15] Rupesinghe N.L., Chhowalla M., Teo K.B.K., Amaratunga G.A.J. // J. Vac. Sci. Technol. B. 2003. Vol. 21. P. 1071-1076.

[16] Eletskii A.V. // Adv. Phys. Sci. 2010. Vol. 53. P. 863-892.

[17] Bocharov G.S., Eletskii A.V. // Nanomaterials. 2013. Vol. 3. P. 393-442.

[18] Murphy E.L., Good R.H. // Phys. Rev. 1956. Vol. 102. N 6. P. 1464-1473.

[19] Mayer A., Lambin Ph. // Carbon. 2002. Vol. 40. P. 429-436.

[20] Sun J.P., Zhang Z.X., Hou S.M., Zhang G.M. // Appl. Phys. A. 2002. Vol. 75. P. 479-483.

[21] Vincent P., Purcell S.T., Journe C., Binh V.T. // Phys. Rev. B. 2002. Vol. 66. P. 075406.

[22] Vul' A.Ya., Reich K., Eidelman E., Terranova M.L., Ciorba A., Orlanducci S., Sessa V., Rossi M. // Adv. Sci. Lett. 2010. Vol. 3. N 2. P. $110-116$.

[23] Бабенко А.Ю., Дидейкин А.Т., Эйдельман Е.Д. // ФТТ. 2009. Т. 51. Вып. 2. С. 410-414.

[24] Лакалин А.В., Павлов А.А., Шаманаев А.А. // Микроэлектроника. 2017. Т. 46. Вып. 1. С. 14-20.

[25] Бочаров Г.С., Елечкий А.В. // ЖТФ. 2007. Т. 77. Вып. 4. C. $107-112$.

[26] Бочаров Г.С., Елещкий А.В., Sommerer T.J. // ЖТФ. 2011. Т. 81. Вып. 4. С. 111-116.

[27] Займан Джс. Электроны и фононы. М.: Изд-во иностранной литературы, 1962. 488 с. (Ziman J.M. Electrons and phonons. Oxford Clarendon press, 1960).

[28] Hone J., Llaguno M.C., Biercuk M.J., Johnson A.T., Batlogg B., Benes Z., Fischer J.E. // Appl. Phys. A. 2002. Vol. 74. P. 339-343. 